\title{
Quantum Chromodynamics on the Lattice 2009
}

\author{
Akira Ukawa \\ University of Tsukuba, 1-1-1 Tennodai, Tsukuba, Ibaraki 305-8577, Japan
}

\begin{abstract}
Lattice QCD is entering a new era as a theoretical machinery to elucidate the physics of strong interactions. We review the algorithmic progress over the past decade behind this advance, and the physics progress powered by it emphasizing flavor physics most relevant for the Lepton Photon Conference series.
\end{abstract}

\section{Introduction}

Lattice QCD has been turning a corner in the last couple of years since the time of LeptonPhoton 2007 [1]. Previously, despite the premise, it remained an approximate method requiring extrapolations in a number of ways to extract physical information. For example, "quenching" the quark determinant ignored the vacuum polarization effects of quarks, and unphysically large values of quark masses in the simulations required help from phenomenological models to estimate values for the physical quark masses.

Progress over years has been removing these restrictions one by one. Most recently, a class of algorithms has been developed which, coupled with deepened understanding of the dynamics of gluon and quark fields, has enabled the reduction of up and down quark masses almost down to the physical point of a few MeV. Thus, lattice QCD is becoming a real first principle method, not only in principle but also in practice, for calculating physical quantities directly at the physical point.

We begin this review with a brief description of the recent algorithmic progress. We next describe the status of lattice studies of flavor physics. Reflecting the algorithmic progress, these studies are increasingly based on the physical point simulations. The topics center around the precision determination of the Cabibbo-Kobayashi-Maskawa mixing matrix, and the light and heavy quark quantities relevant for this task. We shall also make a sojourn into thermodynamics of QCD matter and discuss high density region of QCD; there has been some potentially interesting progress reported this summer.

Lattice QCD in research style has much in common with high energy accelerator experiment; supercomputer installations are costly and need to be planned well ahead, and research groups, either small or large, are generally needed to secure necessary supercomputers time allocations. In particular, generation of gluon configurations is an expensive affair both in terms of computing resources and researcher power. Once generated, however, they can be exploited in more than one ways to tap physical information buried in them. The international lattice QCD community has been working since the summe of 2002 to develop an international data grid infrastructure so that researchers worldwide can benefit from generated configurations. We briefly describe this activity before concluding this review. 


\section{Going to the Physical Point}

Lattice QCD calculations have to deal with four systematic errors, namely, (i) inclusion of quark vacuum polarization effects, (ii) small enough lattice spacing, (iii) large enough lattice size, and (iv) physically light values for the up, down and strange quarks. Of these four, the quenching approximation which ignores (i) became obsolete around 2000 due to the development of dynamical quark algorithms and computer power. Simulations including a degenerate pair of up and down quarks, and a heavier strange quark, often dubbed $N_{f}=2+1$ simulations, have become routine since then. For (ii), a variety of improvement schemes have been developed, with a variable degree of success, and are embodied in today's simulations in one way or another. In essence one adjusts the form of the lattice action and operators for

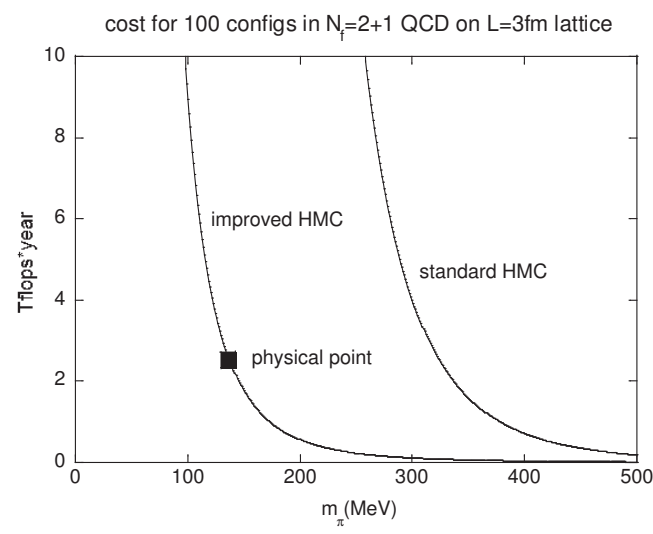

Figure 1: Computational cost for generating 100 independent gluon configurations with the conventional and improved HMC algorithm for $N_{f}=2+1$ QCD with the Wilson-clover quark action. observables by terms higher order in the lattice spacing so that the finite lattice spacing errors in the physical observables are reduced as much as possible. Against (iii) we have had no remedy other than to use large enough volume, relying on increase of computing power. Finally the issue (iv) of using the light enough quark masses has been the most difficult computational problem in lattice QCD. Until quite recently lattice QCD simulations had to be run at the pion mass as heavy as $500 \mathrm{MeV}$, and effective theories such as chiral perturbation theory have to be evoked to carry out a long and unreliable extrapolation to the physical point with $m_{\pi}=135 \mathrm{MeV}$.

The reason behind this difficulty is the necessity to invert the lattice Dirac operator $D$. The computational cost for the inversion increases as the inverse of the minimum eigenvalue, i.e., the quark mass, and so $1 / m$. In the hybrid Monte Carlo algorithm, which is standard for dynamical quark simulations, this inversion is carried out $1 / \delta \tau \propto 1 / m$ times for every gluon configuration generated. Hence the computational cost blows up at least as fast as $1 / \mathrm{m}^{2}$ for small quark masses, and more like $1 / \mathrm{m}^{3}$ if one includes autocorrelation between successive configurations. In Figure 1 the solid line on the right shows an estimate in 2001 [2] for generating 100 independent gluon configurations for a lattice of physical size $L=3 \mathrm{fm}$ at the lattice spacing $a=0.1 \mathrm{fm}$ using the standard hybrid Monte Carlo algorithm in $N_{f}=2+1$ lattice QCD with the Wilson-clover quark action. The computational cost soars far above the 10 Tflops*year mark well before the physical pion mass. The figure shows that even if one uses a computer which executes the lattice QCD code at the speed of 10Tflops, a full 1 year is needed just to do simulations at an unphysically heavy pion of $260 \mathrm{MeV}$.

The recent progress came from the realization that the magnitude of gluon and quark contributions to the force term in the hybrid Monte Carlo algorithm is stratified [3]: the gluon contribution $F_{g}$ has the largest magnitude and has short-range contributions only, next comes in magnitude the short-range contributions of the quark force $F_{q}^{U V}$, and finally the long-distance part of the quark force $F_{q}^{I R}$. There is a clear separation in the magnitude of the three terms, 


\begin{tabular}{|llllll|}
\hline Collaboration & quark action & $a(\mathrm{fm})$ & $m_{\pi} L$ & $m_{\pi}(\mathrm{MeV})$ & ref \\
\hline MILC & staggered & 0.06 & 4.0 & 180 & {$[6]$} \\
PACS-CS & wilson-clover & 0.09 & 2.3 & 155 & {$[7]$} \\
BMW & wilson-clover & 0.09 & 4.0 & 190 & {$[8]$} \\
RBC-UKQCD & domain-wall & 0.09 & 4.0 & 290 & {$[9]$} \\
JLQCD & overlap & 0.11 & 2.8 & 320 & {$[10]$} \\
ETM & twisted mass $\left(N_{f}=2\right)$ & 0.07 & 3.0 & 250 & {$[11]$} \\
\hline
\end{tabular}

Table 1: Recent large-scale $N_{f}=2+1$ simulations.

typically of order $\left\|F_{g}\right\|:\left\|F_{q}^{U V}\right\|:\left\|F_{q}^{I R}\right\| \approx 25: 5: 1$ in simulations done today. In such a situation, one can invoke a multi-time step evolution in the hybrid Monte Carlo algorithm using different step sizes for the three force terms in proportion of $\delta \tau_{g}: \delta \tau_{q}^{U V}: \delta \tau_{q}^{I R} \approx 1$ : $5: 25$. In the standard single time step algorithm, one uses the smallest step size $\delta \tau_{g}$ as the common step size for all three forces. Since the inversion of the lattice Dirac operator is computationally dominated by the long-distance part, the multi-time step evolution can speed up the computation roughly by a factor $\delta \tau_{q}^{I R} / \delta \tau_{g} \approx 25$ simply because the number of Dirac inversions is reduced by this factor.

Concrete implementation of the algorithm has a variety of forms. One way to separate the ultraviolet and infrared quark modes is the Schwarz domain decomposition [3]. An alternative is to apply the idea of mass preconditioner [4] to the quark force in the hybrid Molecular dynamics [5]. The solid line on the left in Figure 1 shows the cost for domain-decomposed hybrid Monte Carlo algorithm [7] as compared to that for the standard algorithm. Clearly, reaching the physical point has become reality with 10 Tflops-class computers for the lattice parameters given.

In Table 1 we list the parameters of representative large-scale simulations pursued today. Except the last entry from the ETM Collaboration using the twisted mass formalism, these are all $N_{f}=2+1$ simulations fully incorporating dynamical effects of up, down, and strange sea quarks. They utilize the algorithmic progress described above so that the pion mass is reaching down to $m_{\pi} \approx 200-300 \mathrm{MeV}$. There has even been a conscious attempt by the PACS-CS Collaboration to reach the physical point at $m_{\pi}=135 \mathrm{MeV}$, and this trend is accelerating.

We emphasize that this progress is pushing lattice QCD to an entirely new stage. The quark mass dependence of physical observables is difficult to control because of potential logarithmic singularities reflecting the infrared divergences at the chiral limit. With the possibility of making calculations directly with the physical pion mass, we no longer have to worry about this issue. In addition, there certainly is esthetic appeal in the ability to work with the physical pion mass since we shall no longer be simulating but actually calculating the strong interaction as it is taking place in Nature.

\section{Fundamental Constants}

The values of quark masses are one of the fundamental quantities of Nature which require lattice QCD to pin down. Accurate control of the behavior of hadron masses at physically small up and down quark masses as well as that of renormalization factors is required for a reliable calculation here. Recent advance of $N_{f}=2+1$ simulations toward the physically light pion mass and non-perturbative estimates of renormalization factors help improve those two aspects. 
In the $\overline{M S}$ scheme at $\mu=2 \mathrm{GeV}$, recent $N_{f}=2+1$ data as reviewed in [12] are indicative of a value $\bar{m}=\left(m_{u}+m_{d}\right) / 2 \approx 3 \mathrm{MeV}$ for the average up and down quark and $m_{s} \approx 90 \mathrm{MeV}$ for strange quark.

Another fundamental constant characterizing the strong interaction is the value of the QCD coupling constant. In Figure 2 we show a comparison of $\alpha_{s}^{\bar{M} S}\left(\mu=M_{Z}\right)$ from experimental determinations based on perturbative QCD and from lattice QCD. The experimental average this year [13] is given by $\alpha_{s}^{\bar{M} S}\left(\mu=M_{Z}\right)=0.1186 \pm 0.0011$. The most elaborate lattice determination is from HPQCD whose value in 2008 [14] has been updated this year to $\alpha_{s}^{\bar{M} S}\left(\mu=M_{Z}\right)=0.1184 \pm$ $0.0004[15]$. Again the lattice value is based on $N_{f}=2+1$ simulations, continuum extrapolated, and includes estimate of systematic errors. The agreement is quite remarkable, and attests to the fact that the single coupling of QCD describes the dynamics of strong interaction from ultraviolet to infrared scales.

\section{Exploring Flavor Physics}

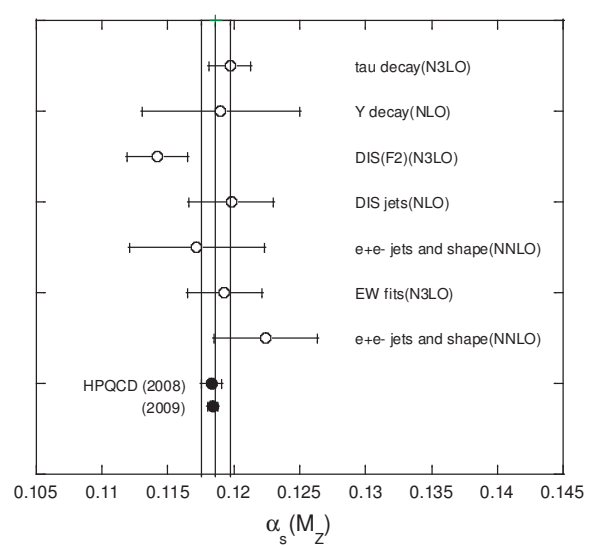

Figure 2: QCD coupling constant as determined from experiment and lattice QCD. The vertical band is the 2009 experimental average [13].

\subsection{Constraints on the Cabibbo-Kobayashi-Maskawa Matrix elements}

\begin{tabular}{|lll|}
\hline quantity & value & error \\
\hline$\hat{B}_{K}$ & $0.725 \pm 0.028$ & $4 \%$ \\
$\xi$ & $1.243 \pm 0.028$ & $2 \%$ \\
$\left|V_{u b}\right|_{\text {excl }}$ & $3.42 \pm 0.37 \times 10^{-3}$ & $11 \%$ \\
$\left|V_{c b}\right|_{\text {excl }}$ & $38.6 \pm 1.2 \times 10^{-3}$ & $3 \%$ \\
$f_{K}$ & $155.8 \pm 1.7 \mathrm{MeV}$ & $1 \%$ \\
\hline
\end{tabular}

Table 2: Lattice QCD inputs as of summer 2009 for constraining the CKM matrix as compiled in [16]. Here $\xi=f_{B_{s}} \sqrt{B_{B_{s}}} / f_{B_{d}} \sqrt{B_{B_{d}}}$.

We now make some specific comments.
Application of lattice QCD toward flavor physics has centered around the constraints imposed on the Cabibbo-Kobayashi-Maskawa mixing matrix elements. In Table 2 we quote the values of representative observables relevant for this purpose as reported by Van de Water at the Lattice 2009 Symposium this summer [25]. It is worth noting that these numbers all come from $N_{f}=2+1$ simulations, and that all errors, either statistical or systematic, the latter arising from chiral extrapolations, finite volumes, or finite lattice spacings, were either calculated or estimated.

\subsubsection{Kaon B parameter}

The Kaon box parameter $B_{K}$ enters into the determination of the CKM matrix through the direct $\mathrm{CP}$ violation parameter $\epsilon_{K}$. In Figure 3 we show how lattice determination of $B_{K}$ has progressed over the years. The three points on the left show a tracking through the plenary reports at the annual lattice symposia in the year 1996 [17], 2000 [18], and 2005 [19]. The inner 
error bars show statistical error, and the outer error bars estimates of systematic errors.

The report in 1996 reflects the calculation by the JLQCD Collaboration published next year [20] in which the continuum limit was taken for the first time, albeit in the quenched approximation. The staggered quark action was employed, and a large error is due to a nonlinear dependence on the lattice spacing expected for the staggered action.

The box parameter is particularly sensitive to the chiral property of the underlying lattice action. For this reason it became the first natural target of simulations with the domain wall quark action pursued vigorously by the RBC-UKQCD Collaboration. The first results in quenched QCD came in around 2000 [21, 22], and by 2005 an $N_{f}=2$ estimate with dynamical up and down quarks was available [23].

The two points on the right are the most recent calculations, finally in $N_{f}=2+1$ QCD. The result in 2008 was reported with the domain-wall quark action at a single lattice spacing [24]. This year a new calculation using the overlap fermion formalism on $N_{f}=2+1$ dynamical gluon configurations

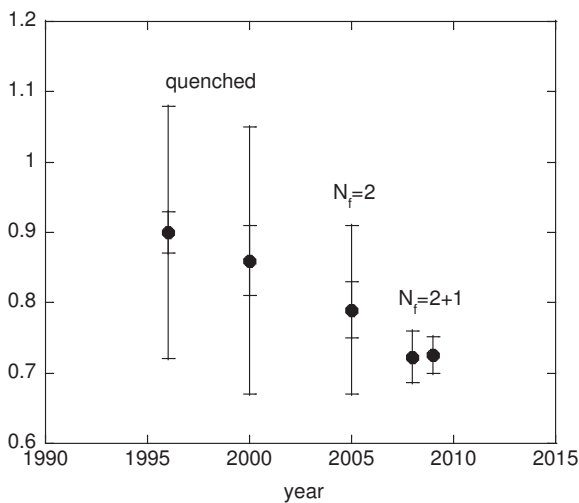

Figure 3: Renormalization group invariant $B_{K}$ over the years. See text for explanation. generated with staggered sea quarks was reported [25]. The 2009 result is continuum extrapolated based on two lattice spacings. The $4 \%$ error in the latest results for $B_{K}$ is smaller than the $10 \%$ error due to $\left|V_{c b}\right|^{4}$ which enters into $\epsilon_{K}$.

\subsubsection{A non-lattice comment on $\epsilon_{K}$}

Buras and Guadagnoli [26] made an important comment on the numerical value connecting the experimental $\epsilon_{K}$ and theoretical $B_{K}$. In the expression

$$
\epsilon_{K}=e^{i \phi_{\epsilon}} \sin \phi_{\epsilon}\left(\frac{\operatorname{Im} M_{12}^{K}}{\delta M_{K}}+\frac{\operatorname{Im} A_{0}}{\operatorname{Re} A_{0}}\right)=\kappa_{\epsilon} \frac{\operatorname{Im} M_{12}^{K}}{\delta M_{K}},
$$

the second term $\operatorname{Im} A_{0} / \operatorname{Re} A_{0}$, being small, is usually neglected. However, with increasingly improved estimates of $B_{K}$ and $V_{c b}$, this correction is significant. Buras and Guadagnoli estimates that

$$
\kappa_{\epsilon} \approx \sqrt{2} \sin \phi_{\epsilon}\left(1-\frac{1}{\omega} \operatorname{Re}\left(\frac{\epsilon_{K}^{\prime}}{\epsilon_{K}}\right)+\frac{1}{\sqrt{2}\left|\epsilon_{K}\right|} \frac{\operatorname{Im} A_{s}}{\operatorname{Re} A_{2}}\right)=0.92 \pm 0.02 .
$$

This implies that the $\epsilon_{K}$ band in the $\rho-\eta$ plane for the CKM matrix might move up by about $10 \%$.

\subsubsection{Inclusive $v s$ exclusive determination of $V_{c b}$ and $V_{u b}$}

The $V_{c b}$ matrix element can be determined by combining the experimental $B \rightarrow D^{*}$ rate with a lattice determination of the corresponding form factor [27], and similarly for the $V_{u b}$ matrix element via the $B \rightarrow \pi$ decay [28]. The lattice numbers have not changed since 2008, and the 
values for $V_{c b}$ and $V_{u b}$ still differ from the estimates [29] combining the inclusive decay rates with non-lattice calculations of decay amplitudes at a two $\sigma$ level. The situation is illustrated in Figure 4.

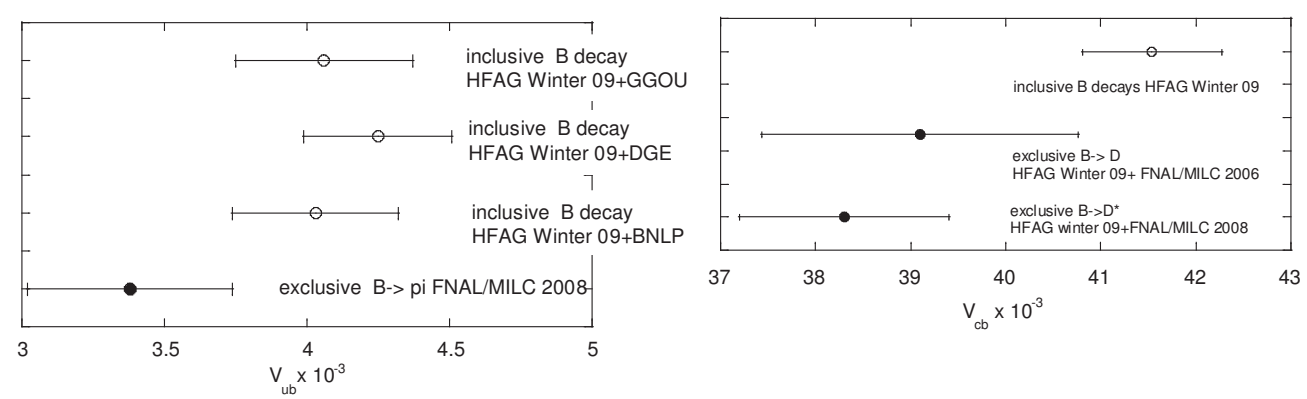

Figure 4: $V_{c b}$ and $V_{u b}$ matrix element as determined from exclusive rates and lattice form factor (filled circles) and inclusive rates and non-lattice transition amplitude estimates (open circles).

\subsubsection{D meson decay constants}

In Figure 5 we compare lattice results for the $D$ and $D_{s}$ meson decay constant against experiment. The latest 2009 experimental numbers from CLEO [30] are $f_{D}=205.8 \pm 8.9 \mathrm{MeV}$ and $f_{D_{s}}=259.5 \pm$ $7.3 \mathrm{MeV}$.

On the lattice, there has been no change in the value from HPQCD Collaboration [31] who uses the HISQ form of the staggered quark action for the charm quark. The discrepancy in $f_{D_{s}}$ still remains.

The estimates from Fermilab-MILC Collaboration who uses the Wilson-clover quark action has been updated this summer [32] due to a $2.3 \%$ revision in the lattice scale in physical units, and the numbers have gone up. Within the relatively large error of about $4 \%$, they are consistent with experiment. Lattice QCD has to resolve the difference between the HISQ and Wilson-clover determinations which is a systematic effect.

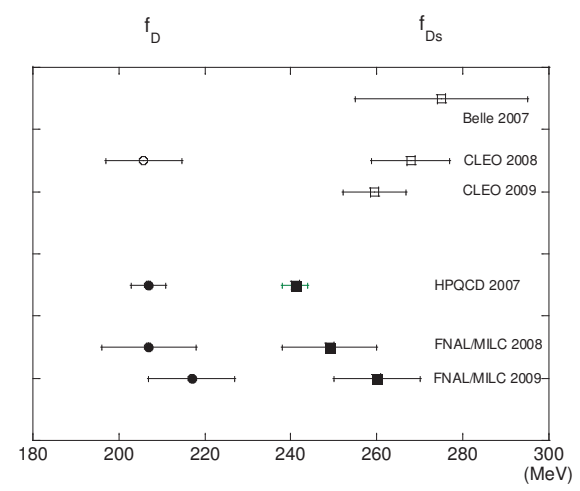

Figure 5: Lattice (filled symbols) and experimental (open symbols) determination of $D$ and $D_{s}$ meson decay constant from 2007 to 2009.

\subsection{CKM unitarity}

In Table 3 we present the status with the unitarity check of the CKM matrix using as much lattice input as possible. For the first row, the very precise value for $V_{u d}$ comes from a nonlattice analysis of the nuclear transition rates [33]. The $V_{u s}$ is calculated from experimental $K \rightarrow \pi$ decay rate and an $N_{f}=2+1$ lattice QCD determination of the form factor [34], and 
$V_{u b}$ is taken from the lattice determination from the exclusive $B \rightarrow \pi$ decay [28] as discussed in Subsection 4.1.3. The first row unitarity holds to within 0.1\%.

\begin{tabular}{|llll|}
\hline$V_{u d}$ & $V_{u s}$ & $V_{u b}$ & $\sum_{j}\left|V_{u j}\right|^{2}-1$ \\
\hline 0.97425 & 0.2246 & 0.00342 & -0.0004 \\
\pm 0.00022 & \pm 0.0012 & \pm 0.00037 & \pm 0.0013 \\
\hline$V_{c d}$ & $V_{c s}$ & $V_{c b}$ & $\sum_{j}\left|V_{c j}\right|^{2}-1$ \\
\hline 0.239 & 0.969 & 0.039 & -0.002 \\
\pm 0.032 & \pm 0.105 & \pm 0.001 & \pm 0.110 \\
\hline
\end{tabular}

Table 3: First and second row unitarity of the CKM matrix spacing $a^{-1} \sim 2 \mathrm{GeV}$ which has been typically used in lattice calculations. In order to control systematic errors coming from $m_{c} a$ being not small, $N_{f}=2+1$ simulations at smaller lattice spacings are required. Large-scale simulations are moving in this direction, so hopefully there will be progress here in the near future.

\subsection{Lattice calculation of $\epsilon^{\prime} / \epsilon$}

Successful calculation of the $\mathrm{CP}$ violation parameter ratio $\epsilon^{\prime} / \epsilon$ has been a major challenge in lattice QCD since the middle of 1980's. Chiral symmetry turned out crucial to control renormalization and large fluctuations of ultraviolet origin in the Penguin contributions. It was only in 2003 that results with meaningful error estimations were obtained with domain-wall QCD [36, 37]. The calculations relied on the rewriting of the two-body $K \rightarrow \pi \pi$ amplitude in terms of the one-body $K \rightarrow \pi$ and $K \rightarrow$ vacuum amplitude to lowest order of chiral perturbation theory. It was quite a disappointment to find that the results do not agree with experiment even in sign. While it was not clear if quenching was the cause or possible failure of chiral perturbation theory in the range of pion mass $m_{\pi} \approx 0.5 \mathrm{MeV}$ is to be blamed, the severe lesson taught us that full QCD calculations directly addressing the two-body decay amplitude is needed.

The theoretical framework for this purpose was laid down in 2001 [38]; one chooses a lattice volume $L^{3}$ such that the $\mathrm{K}$ meson energy $E_{K}(L)$ on this volume matches the two-pion energy at the same volume $E_{\pi \pi}(L)$. One can then prove that the lattice value for the two-body decay amplitude $\left\langle K\left|H_{W}\right| \pi \pi\right\rangle_{\text {lattice }}$ calculated on this volume is proportional to the physical amplitude $\left\langle K\left|H_{W}\right| \pi \pi\right\rangle_{\text {physical }}$ up to a calculable factor.

This framework was applied to the isospin $I=2$ sector last year using the domain wall QCD in the quenched approximation [39]. In this isospin channel, the troublesome Penguin contribution is absent, and previous attempts have already yielded values in agreement with experiment. The advance achieved by the new calculation is the working proof that the finitevolume method works for the $\mathrm{K}$ meson decay.

The calculation in the isospin $I=0$ channel is much harder, but we can expect progress as $N_{f}=2+1$ full QCD calculations are steadily progressing toward large volume and physical pion mass. 


\section{$5 \quad$ Exploring High Density}

Temperature and density are two dials which allow us to explore the dynamics of QCD relevant under the extreme conditions in the Early Universe or in relativistic heavy ion collision experiments. At zero density the phase diagram as a function of temperature and the number of quarks has been studied extensively in the past. Theoretically the order of the transition from hadron gas to quark gluon plasma is sensitively dependent on the up, down and strange quark masses. The most recent calculation in 2006 [40] using finite-size scaling analyses made a strong case that the transition is a crossber at a temperature range $T_{c} \approx 150-170 \mathrm{MeV}$ for the physical quark mass. There has been no major change since this work.

On the other hand, much less is known on the phase diagram of QCD at finite density. This is due to the difficulty, in spite of many attempts, to resolve the sign problem, i.e., the quark determinant for non-zero quark chemical potential $\mu_{q}$ becomes complex, and hence Monte Carlo methods break down. There are indications, based on the reweighting technique [41] and the method of Taylor expansion in $\mu_{q}$ [42] that the crossover behavior at zero density continues into the region of non-zero density but turns into a first-order phase transition through a critical point.

Most simulations for non-zero density have been attempted with the chemical potential formalism, i.e., using the grand partition function. In principle one can equally well use the canonical partition function in which the number of quarks $N_{q}$ or baryon number $n_{B}=N_{q} / 3$ is fixed. The relation between the two partition functions are given by

$$
Z_{\text {grandcanonical }}\left(T, m_{q}, \mu_{B}\right)=\sum_{n_{B}} e^{n_{B} \mu_{B} / T} Z_{\text {canonical }}\left(T, m_{q}, n_{B}\right)
$$

and hence the path integral for the canonical partition function is given by

$$
Z_{\text {canonical }}\left(T, m_{q}, n_{B}\right)=\int[d U]\left[\int_{0}^{2 \pi} d \phi e^{-i 3 n_{B} \phi} \operatorname{det} D\left(U, m_{q}, \mu=i \phi T\right)\right] e^{-S_{\text {gluon }}(U)}
$$

Since the quark determinant is an extensive quantity, it is important to accurately estimate the projection of the quark determinant in this equation. An exact evaluation of the projection [43] is computationally expensive and hence not very practical for large lattices. An interesting idea is to apply saddle point approximation expected to be valid for large spatial volume [44]. A quantitative measure of control of the approximation is needed for this method.

This year a new attempt was made to control the projection [45].. The direct projection has an apparent problem that cancellation of oscillations whose magnitude becomes exponentially large in volume has to be controled. Numerically this is a difficult task, and hence the idea is to carry out the projection to the logarithm of the determinant via

$$
\log \operatorname{det} D\left(U, m_{q}, \mu\right)=\sum_{n_{B}} e^{n_{B} \mu_{B} / T} A\left(U, m_{q}, n_{B}\right)
$$

Figure 6 shows the result [45] for the chemical potential as a function of baryon number (left panel) and the phase diagram (right panel) for $N_{f}=3$ QCD with Wilson quark action on a $6^{3} \times 4$ lattice. The cubic variation on the left panel indicates a first-order phase transition, and the familiar Maxwell construction yields the boundary of the two-phase coexistence region plotted on the right on the $T-\mu$ plane. We see that the coexistence region becomes narrower 

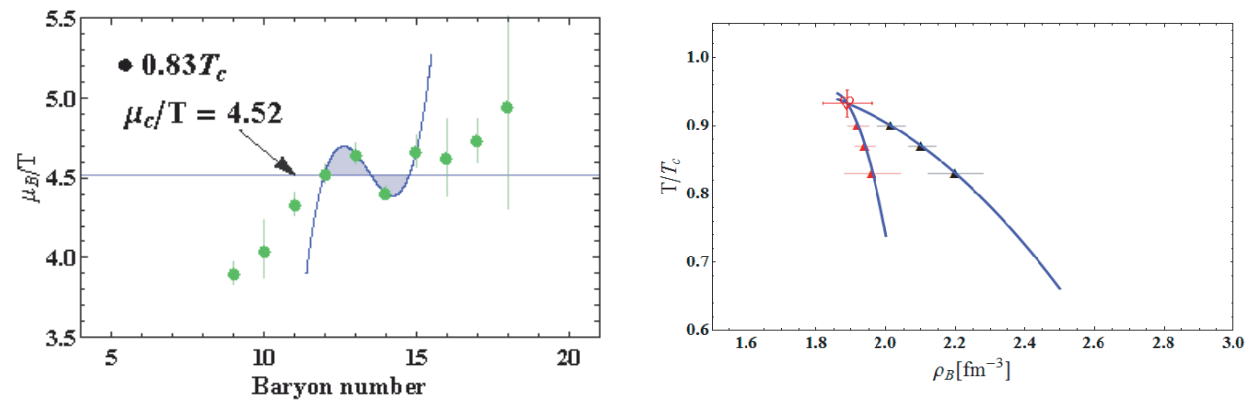

Figure 6: Chemical potential calculated as a function of baryon number (left panel) and the two-phase coexistence region on the $T-\rho_{B}\left(\rho_{B}=n_{B} / V\right)$ plane (right panel) calculated by a microcanonical approach [45].

as the temperature increases, likely terminating at the estimated endpoint marked by an open circle.

It may well be that the sign problem has not manifested itself in this calculation because of the heavy pion mass $\left(m_{\pi} \approx 700 \mathrm{MeV}\right)$ and the small lattice size $\left(6^{3} \times 4\right)$. It is nonetheless an encouraging result and is worth pursuing as an alternative to the grand canonical approach.

\section{Collaborating World-wide}

Lattice QCD is practiced across the globe. There are about a dozen major centers scattered in Japan, Australia, EU countries (including Germany, France, United Kingdom, Italy, and Spain), USA and Canada. As of 2009 the total computing capacity employed for lattice QCD is about half a petaflops, which is about $3 \%$ of the total world high performance computing resources.

The most time consuming element in lattice QCD calculation is the generation of gluon configurations with dynamical quarks. Once generated those configurations can be used by anyone to calculate physical observables of one's interest. Since 2002, this has motivated the lattice QCD community to make an effort toward a world-wide collaboration called International Lattice Data Grid (ILDG) to organize and run a data grid for gluon configurations [46].

ILDG consists of a number of regional grids, each operating on its own. For a world-wide sharing of configurations, a standardized $\mathrm{xml}$ called qcdml has been defined to describe gluon data, and standard middleware has been designed for interoperability of the regional grids through ILDG. After 4 years of preparation, ILDG started service in June 2006 [47]. The number of gluon ensembles (set of configurations) and downloads have been steadily increasing over the years as shown in Figure 7. ILDG has become an established infrastructure in the lattice QCD community, and its role will continue to increase in the years to come.

\section{Conclusions}

Over the last couple of years, realistic calculations directly at the physical point have finally become reality in lattice QCD. This is a fruit of continuous effort over 25 years toward better 

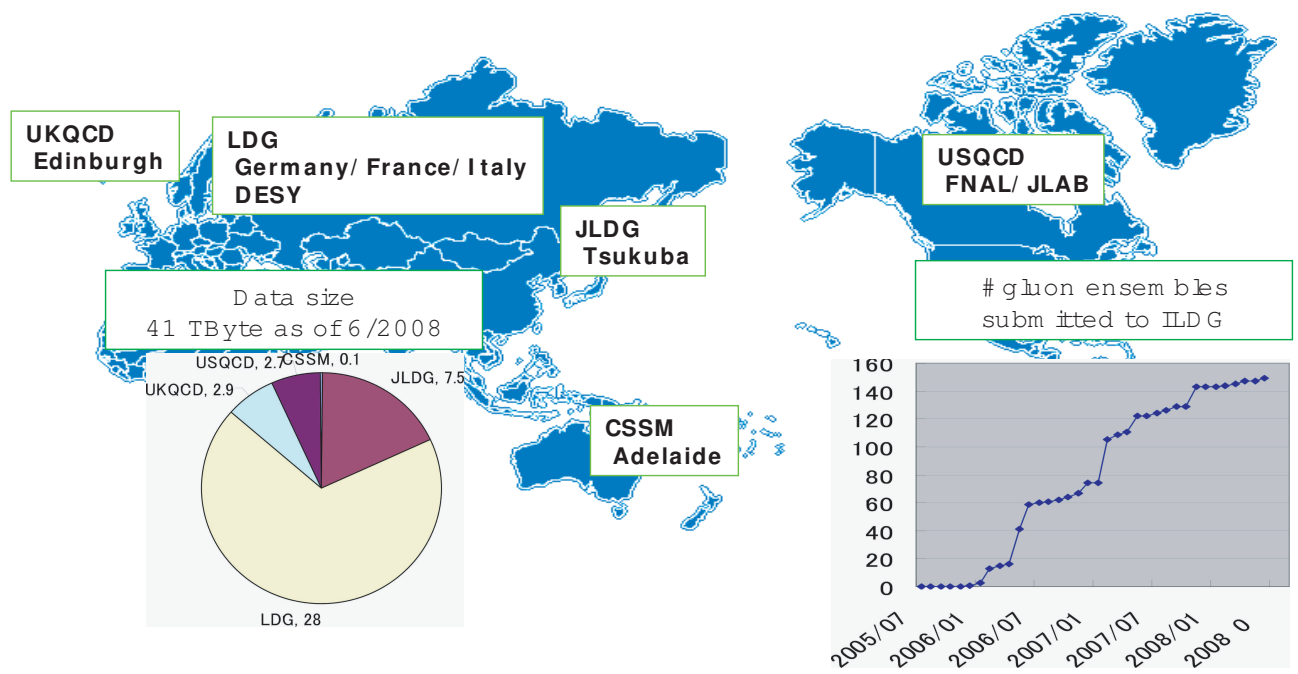

Figure 7: International Lattice Data Grid for a world-wide sharing of gluon configurations.

physics understanding, better algorithms and more powerful computers. Personally I believe that this entails a change of philosophy from "simulation" to "calculation": if lattice spacing is sufficiently small, there are no more approximations or extrapolations, and gluon configurations produced on the computer is strong interaction in Nature itself.

Armed with this tool, I expect that the fundamental issues of lattice QCD as particle theory make major progress over the next five-year range. Those include single hadron properties and fundamental constants, precision flavor physics with errors bound below $1 \%$ level and resolution of old issues such as $K \rightarrow \pi \pi$ decays, and hot/dense QCD explored with chiral lattice action on large lattices.

And there lies beyond the vast area of multi-hadron systems and atomic nuclei for our nuclear physics colleagues to explore, e.g., nuclear force from lattice QCD, exotic nuclei with unusual neutron/proton ratios and/or strangeness, and element synthesis in supernovae and so on.

\section{Acknowledgments}

I would like to thank Christopher Aubin, Christine Davies, Phillipe de Forcrand, Shoji Hashimoto, Taku Izbuchi, Chulwoo Jung, Kazuyuki Kanaya, Andreas Kronfeld, Mikko Laine, An-yi Li, Vittorio Lubicz, Tetsuya Onogi, Dirk Pleiter, Enno Scholz, James Simone, Doug Toussaint, and Ruth Van de Water for sharing their expertise with me.

This research is supported in part by the Grant-in-Aid (No. 18104005) of Ministry of Education, Culture, Science and Sports of Japan.

\section{References}

[1] C. T. H. Davies, Proceedings of Lepton-Photon 2007, arXiV:0807.1402 (hep-ph)(2007). 
[2] A. Ukawa, Nucl. Phys. B(Proc. Suppl.), 106, 195 (2002).

[3] M. Lüscher, Comput. Phys. Commun., 165, 199 (2005).

[4] M. Hasenbusch, Phys. Lett. B 519, 177 (2001).

[5] C. Urbach, K. Jansen, A. Shindler, U. Wenger, Comput. Phys. Commun. 174, 87 (2006).

[6] MILC Collaboration, A. Bazavov et al., arXiv:0903.3598 (2009).

[7] CP-PACS Collabortion, S. Aoki et al, Phys. Rev. D 79, 034503 (2009).

[8] S. Durr et al., Science, 322, 1224 (2008).

[9] RBC and UKQCD Collaboration, R. Mawhinney et al., arXiv:0910.3194 (2009).

[10] JLQCD Collaboration, H. Fukaya et al., Phys. Rev. Lett. 104, 122002 (2010).

[11] ETM Collaboration, C. Alexandrou et al., Phys. Rev. D 78, 014509 (2008).

[12] E. Scholz, Plenary talk at Lattice 2009, arXiv:0911.2191 (2009).

[13] S. Bethke, arXiv:0908.1135 (2009).

[14] HPQCD Collaboration, C. T. H. Davies et al., Phys. Rev. D 78, 114507 (2008).

[15] C. T. H. Davies, private communication (2009).

[16] R. Van de Water, Plenary talk at Lattice 2009; see, for details, J. Laiho, E. Lunghi, R. Van de Water, Phys. Rev. D 81, 034503 (2010).

[17] S. Sharpe, at Lattice 1996, Nucl. Phys. B(Proc. Suppl.) 53, 181 (1997)

[18] C. Lellouch, at Lattice 2000, Nucl. Phys. B(Proc. Suppl.) 94, 142 (2001)

[19] C. Dawson, at Lattice 2005, PoS LAT2005, 007 (2006).

[20] JLQCD Collaboration, S. Aoki et al., Phys. Rev. Lett. 80, 5271 (1998).

[21] RBC Collaboration, Phys. Rev. D 68, 114506 (2003).

[22] CP-PACS Collaboration, S. Aoki et al, Phys. Rev. D 64, 114506 (2001).

[23] RBC Collaboration, Y. Aoki et al., Phys. Rev. D 72, 114505 (2005).

[24] RBC and UKQCD Collaboration, Phys. Rev. Lett. 100, 0321001 (2008)

[25] C. Aubin, J. Laiho, R. Van de Water, Phys. Rev. D 81, 014507 (2010).

[26] A. Buras and D. Guadagnoli, Phys. Rev. D 78, 033005 (2008).

[27] Fermilab Lattice and MILC Collaborations, C. Bernarad et al, Phys. Rev. D 79, 014506 (2009). See also M. Okamoto et al, Nucl. Phys. B (Proc. Suppl.) 140, 461 (2005)

[28] Fermilab Lattice and MILC Collaborations, J. A. Bailey et al, Phys. Rev. D 79, 054507 (2009). 
[29] Heavy Flavor Averaging Group, http://www.slac.stadoford.edu/xorg/hfag/semi/ (2009).

[30] CLEO Collaboration, Liming Zhang, arXiv:0810.2328 (2009); CLEO Collaboration, B. I. Eisenstein et al., Phys. Rev. D 78, 052003 (2008).

[31] E. Follana et al, Phys. Rev. Lett. 100, 062002 (2008).

[32] A. Bazavov et al., PoS LAT2009, 249 (2009).

[33] J. C. Hardy and I. S. Towner, Phys. Rev. C 79, 055502 (2009).

[34] P. Bopyle et al., Phys. Rev. Lett. 100, 141601 (2008).

[35] Fermilab Lattice, MILC, and HPQCD Collaborations, C. Aubin et al., Phys. Rev. Lett. 94,11601 (2005).

[36] T. Blum et al., Phys. Rev. D 68, 114506 (2003).

[37] CP-PACS Collaboration, J. Noaki et al/, Phys. Rev. D 68, 14501 (2003).

[38] L. Lellouch and M. Lüscher, Commun. Math. Phys. 219, 31 (2001).

[39] T. Yamazaki, Phys. Rev. D 79, 094506 (2009).

[40] Y. Aoki et al., Nature 443, 675 (2006).

[41] Z. Fodor and S. Katz, JHEP 0404, 050 (2004).

[42] C. Allton et al., Phys. Rev. D 71, 054508 (2005).

[43] P. de Forcrand and S. Kratochivila, Nucl. Phys. B (Proc. Suppl.) 153, 62 (2006).

[44] S. Ejiri, Phys. Rev. D 78, 074507 (2009).

[45] Anyi Li, talk at Lattice 2009, arXiv:1002.4459 (2009).

[46] C. T. H. Davies et al., Nucl. Phys. B (Proc. Suppl.) 119, 225 (2003).

[47] http://ildg.sasr.edu.au/Plone 


\section{Discussion}

Bruno Stella (Universita Roma Tre): I think for the first time the rapporteur of Lattice QCD does not mention glueballs. Why? Could you say something more?

Answer: I do not think there has been substantial progress in recent years, especially on the difficult issue of a reliable estimate of mixing of pure glue states with quark states. Full QCD simulations necessary for this has not reached sufficient statistics so far.

Guido Altarelli (Roma III and CERN): Concerning the discrepancies between the lattice determinations of $f_{D_{s}}$ and the data,is the smallness of the quoted error by HPQCD really justified, also in view of the FNAL/MILC more recent central values and quoted errors?

Answer: This is a difficult question to answer. I suppose they have a large number of data points in quark masses and lattice spacings to estimate the value for physical quark masses and the continuum limit, which helps reduce the error. Their light quark mass also goes down to a fairly small value so that the ambiguity of chiral extrapolation is smaller.

Markus Wobisch (FNAL): You mentioned the possibility of computing properties of hadrons. Could you also compute the distributions of partons inside hadrons?

Answer: Yes, lattice methods can compute the moments of structure functions. The computations becomes progressively difficult for higher moments, however, so only several moments are feasible so far.

Vera Lüth (SLAC): Current lattice calculations are $N_{F}=2+1$. Can we hope to extend calculations to include charm and eventually beauty? At present the dominant error on $V_{u b}$ determinations are quark masses, especially $m_{b}$.

Answer: Dynamical charm is already withing the scope of full QCD simulations. Dynamical beauty is still a future problem since the lattice spacing of larger than $5 \mathrm{GeV}$ necessary for this requires larger than petaflops scale computing resources. However, the estimate of $m_{b}$ will not be affected much by dynamical beauty effects since the $\mathrm{b}$ quark is much heavier than the typical QCD scale.

Bennie Ward (Baylor University): In your plot of the hadron spectrum you only show a small subset of what is in the PDG. Are you cherry-picking? Can you predict glueballs, hybrids, molecules, pentaquarks, etc?

Answer: Confirming the ground state spectrum, though a small subset of PDG, is a basic step to establish the validity of QCD and the predictive capability of lattice QCD. Predictions of other states including exotics will come step by step.

Ahmed Ali (DESY): This question concerns chiral extrapolation on the lattice. Are the pion mass and the light quark masses for which lattice simulations are currently being done at a stage that the chiral perturbation theory can be checked in the sense that some of the constants in chiral pert. theory are now determined by lattice?

Answer: Yes, the low energy constants such as $l_{3}$ and $l_{4}$ have been determined with 
reasonable precision. Further the convergence of ChPT has been examined. At present $\mathrm{SU}(3) \mathrm{ChPT}$ does not seem to be convergent at the physical strange quark mass, while SU(2) ChPT seems to be convergent for physical up-down quark mass in the pseudoscalar sector. The situation may depend on the quantity. 